\title{
RR Lyrae Variables in NGC 7006
}

\author{
Amelia Wehlau, Robert W. Slawson \\ Department of Astronomy, The University of Western Ontario, London, \\ Ontario, CANADA, N6A $3 K^{\prime}$
}

James M. Nemec

Department of Physics and Astronomy, York University, 4700 Keele Street, North York, Onatrio, CANADA, M3J1P3

\author{
Steve Butterworth \\ Department of Physics and Astronomy, McMaster University, \\ Hamilton, Ontario, CANADA, L8S $4 M 1$
}

\begin{abstract}
B and V CCD frames of the globular cluster NGC 7006 have been obtained to study the period change rates and determine photometric colours for the RR Lyr variables.
\end{abstract}

NGC 7006 is a galactic globular cluster at a heliocentric distance of $40 \mathrm{kpc}$ known to contain at least 60 RR Lyrae stars. It was also one of the first clusters studied whose colour-magnitude diagram showed evidence of a "second parameter". Magnitudes of the variable stars were obtained by Rosino and his colleagues from plates taken by Hubble in the 1930's and by Sandage in the 1950's and used to determine periods for many of the variables. These earlier observations were combined with magnitudes from plates taken by Nemec in 1984 and used by Wehlau et al. (1992) to derive period change rates. Statistically significant evidence was found for a radial gradient in these rates, more negative rates being found in the outer region of the cluster.

B and V CCD frames of the cluster were obtained by Nemec in 1984, 1987, and 1989 using the Palomar $1.5 \mathrm{~m}$ telescope and in 1994 with the $1.8 \mathrm{~m}$ telescope of the Dominion Astrophysical Observatory. Most of the frames have now been reduced using magnitude calibrations based on the photometry of Buonanno et al. (1991).

These new observations made it possible to derive periods for some stars for which we were unable to obtain periods earlier. Light curves for four of these stars are shown in Fig. 1. Variables $24(P=0.626277)$ and $27(P=0.567215)$ are each blended with a close companion so that no periods could be obtained earlier. The light curve for V28 $(P=0.497308)$ also represents a new period since the period of 0.56 day given by Pinto \& Rosino (1973) does not fit our data. However this new period which is very close to half a day does fit their observations as well as ours. V76 $(P=0.549564)$ was discovered on the 1984 photographic plates which were taken during a 10 day observing run. 


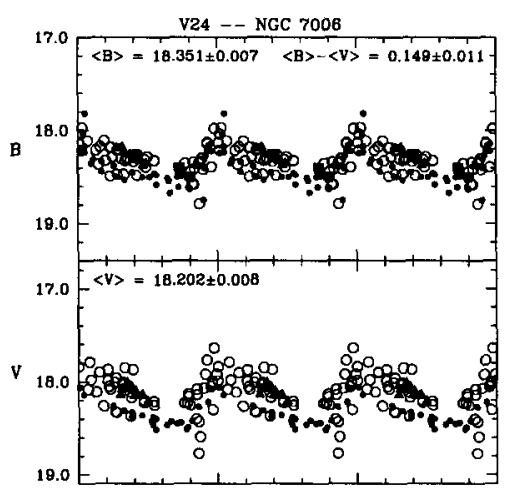

$\phi$



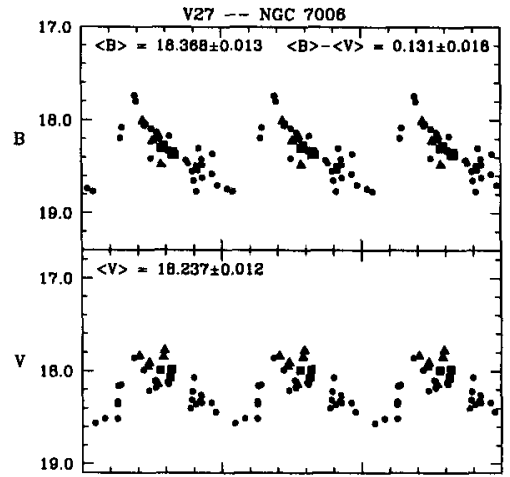

$\phi$

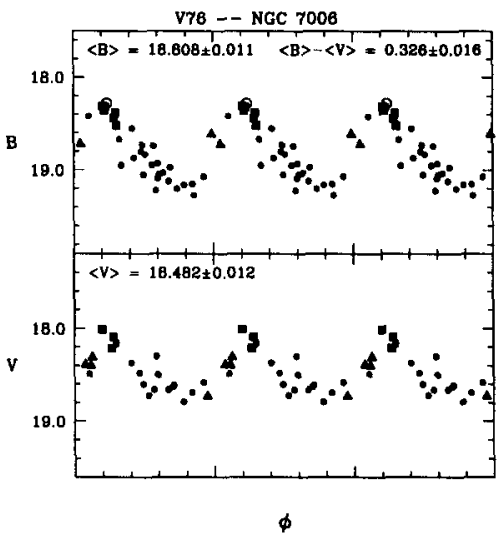

Figure 1. Light curves for V24, V27, V28, and V76 with new periods. The various symbols represent observations in different years.

\section{References}

Buonanno, R., Fusi Pecci, F., Cappellaro, E., Ortolani, S., Richtler, T., \& Geyer, E. H. 1991, AJ, 102, 1005

Pinto, G., \& Rosino, L. 1973, MSAIt, 44, 255

Wehlau, A., Nemec, J. M., Hanlan, P., \& Rich, R. M. 1992, AJ, 103, 1583 